\title{
La quête du vernaculaire dans l'étude de la variation grammaticale
}

\author{
Aydan Coveney \\ Université of Exeter (Royaume-Uni) \\ A.B.Coveney@exeter.ac.uk
}

Résumé. La sociolinguistique variationniste accorde une place privilégiée au style vernaculaire, défini comme le style où l'on accorde le minimum d'attention à la surveillance de son propre discours : d'abord parce que c'est la variété la plus régulière et systématique, n'étant pas susceptible à l'hypercorrection trouvée dans des styles plus formels ; et ensuite puisque c'est dans le style vernaculaire qu'apparaissent pour la première fois de nombreuses innovations linguistiques, et que ce style donne ainsi l'indication la plus fiable de l'état actuel de la langue, dans des perspectives historique et typologique. Lorsqu'on compare deux échantillons de discours non vernaculaire, il faut envisager la possibilité que les deux groupes de locuteurs aient parlé dans deux styles différents, plus ou moins distants du vernaculaire. Alors que le tutoiement n'entraine pas nécessairement l'utilisation du style vernaculaire (l'emploi par un individu de son niveau maximal des variantes informelles à sa disposition), il semble bien être une condition nécessaire pour l'émergence de ce style. Nous émettons donc l'hypothèse que le vernaculaire est pragmatiquement incongru (et inexistant) aux côtés du vouvoiement dans le même discours. Or, dans plusieurs corpus d'entretiens sociolinguistiques, le vouvoiement est majoritaire, voire systématique, et la prudence est donc de mise lorsqu'il s'agit de comparer la variation dans ces corpus. D'ailleurs la dynamique interpersonnelle de l'entretien influe directement sur l'usage de certains domaines grammaticaux, comme les pronoms personnels indéfinis et les structures syntaxiques associées aux actes de parole directifs et expressifs, notamment les interrogatives directes et les phrases négatives emphatiques. Finalement nous proposons un " paradoxe variationniste » (en parallèle avec le célèbre «paradoxe de l'observateur ») : dans la plupart des recherches variationnistes, l'objectif idéal est d'enregistrer, en un temps limité, une quantité importante de discours en style vernaculaire chez des locuteurs représentatifs de la communauté linguistique. Le contexte le plus pratique pour obtenir ces données auprès d'un tel échantillon de locuteurs est l'entretien sociolinguistique, qui, par définition, implique deux inconnus et qui, par conséquent, exclut le style vernaculaire. Ce paradoxe peut être résolu grâce à l'autoenregistrement ou aux conversations de groupes de pairs.

\begin{abstract}
Variationist sociolinguistics often accords pride of place to the vernacular style, defined as the style in which minimal attention is paid to the monitoring of one's own speech: firstly because it is the most systematic and regular variety, being immune from the hypercorrection found in more formal styles; and secondly because it is in the vernacular that many linguistic innovations first appear, and this style thus gives the most reliable indication of the current state of the language, from historical and typological perspectives. When comparing two samples of non-vernacular speech, we must consider the possibility that the two groups of speakers have spoken in two different styles, more or less distant from the vernacular.

Whilst tutoiement does not necessarily entail the use of the vernacular (i.e. the use by an individual of the maximum level of informal variants at their disposal), it does seem to be a necessary condition for the emergence of this style. We therefore hypothesize that the vernacular is pragmatically incongruous (and non-existent) alongside vouvoiement in the same discourse. However in several corpora of sociolinguistic interviews, vouvoiement is the majority address form, and is sometimes even categorical, and therefore caution is necessary when comparing
\end{abstract}


variation in different corpora. Moreover the interpersonal dynamics of the interview directly affect the use of certain grammatical features such as indefinite personal pronouns and syntactic structures associated with directive and expressive speech acts, including direct interrogatives and emphatic negation. Finally we propose a "variationist paradox" (in parallel with the famous "observer's paradox"): in most variationist research, the ideal goal is to record, in a limited time, a significant amount of vernacular speech from a representative sample of the speech community. The most practical context in which to obtain this type of data from such a sample of speakers is the sociolinguistic interview, which, by definition, involves two strangers and therefore excludes the vernacular style. This paradox can be resolved through self-recording or peer-group conversations.

\section{Introduction}

De nombreux observateurs considèrent la parole spontanée informelle comme le type de discours le plus « authentique » ou " écologique », en ce sens qu'il représente la façon dont les gens parlent la plupart du temps, dans des situations non institutionnelles de tous les jours. Cette conviction largement répandue est probablement l'un des facteurs qui ont conduit William Labov à développer le concept du vernaculaire comme « le style où l'on accorde le minimum d'attention à la surveillance de son propre discours » $(1976: 289)$. Dans le courant variationniste, on croit également que le vernaculaire est acquis au cours de la préadolescence, et que d'autres styles, plus formels, y sont superposés vers la fin de l'adolescence et pendant l'âge adulte (cf. Poplack \& StAmand, 2007). Bien entendu, ce sens du terme "vernaculaire " est une extension de son utilisation plus générale en linguistique, pour signifier une variété indigène qui n'a pas été standardisée, et qui est parlée dans une communauté géographiquement définie, que ce soit une ville, une région ou un pays.

Bien que le concept du style vernaculaire soit maintenant fermement établi en sociolinguistique, certains semblent douter de son existence réelle. Selon James Milroy, il s'agit d'une « idéalisation - sur un pied d'égalité avec d'autres idéalisations, comme le dialecte ou la communauté linguistique - et en tant qu'idéalisation il doit être inaccessible dans la pratique » $(1992: 66)^{1}$. Milroy semble impliquer ainsi que le vernaculaire n'existe pas dans la réalité. Toutefois, si l'on considère une définition du style vernaculaire en termes de l'utilisation de variantes (de variables sociolinguistiques), il est en effet possible d'envisager des passages réels de discours qui peuvent être identifiés comme exemples du style vernaculaire. Il existe très probablement, dans une communauté linguistique donnée, certains locuteurs qui, dans certaines situations, utilisent catégoriquement les variantes non standard (ou informelles) de toutes les variables sociolinguistiques à leur disposition. ${ }^{2} \mathrm{Si}$ un tel discours est bien parfois produit en réalité, l'on doit sûrement le qualifier de «style vernaculaire », car il ne pourrait pas être plus informel, en termes quantitatifs.

De toute évidence, de nombreux locuteurs n'emploient pas les variantes informelles ou non standard de manière catégorique, même dans leur discours le plus informel. Il semble néanmoins raisonnable de dire que ces personnes possèdent eux aussi un style vernaculaire, même s'il est moins nettement défini en termes quantitatifs que ce qui est le cas pour ceux qui utilisent catégoriquement des variantes non standard ou informelles. Dans le cadre du travail sur le terrain à long terme dans une communauté donnée, l'on pourrait envisager d'enregistrer et d'analyser à plusieurs reprises des échantillons du discours informel des individus, afin de tracer leur emploi maximal des variantes non standard (ou informelles), et ainsi d'identifier le niveau statistique de leur style vernaculaire. Il faut conclure par conséquent que la notion de « style vernaculaire » est bien valide, et que ce style existe bel et bien en réalité et peut être observé. Qui plus est, il est primordial, lorsque l'on examine des échantillons enregistrés de la langue parlée, de les situer par rapport au style vernaculaire des personnes qui les ont produits. Nous verrons plus tard dans cet article dans quelle mesure différents corpus sociolinguistiques de français parlé représentent un style proche du vernaculaire.

À l'origine, la définition labovienne du vernaculaire comme «le style d'attention minimale » reposait sur l'idée que les styles de parole forment un continuum en corrélation étroite avec le degré d'attention prêtée par le locuteur à son langage pendant l'acte de parler. Étant donné que cette conception de la variation stylistique a été largement supplantée, aux yeux de la plupart des variationnistes, par une théorie plus satisfaisante, celle de «la conception du style en fonction de son auditoire $»^{3}$ (Bell, 1984, 2001), il convient de demander comment le vernaculaire peut être interprété en termes de cette approche. Bell et d'autres sociolinguistes (par exemple, Coupland, 1980 ; Trudgill, 1986) ont démontré que le facteur principal motivant tel ou tel style de parole, après l'identité même du locuteur, est généralement la nature de l'auditoire, et en particulier l'identité de l'allocutaire (ou des allocutaires), et comment le locuteur perçoit cet individu et son langage. La théorie de Bell ne semble pas prévoir différents types d'allocutaires, mais il semble raisonnable d'affirmer que le vernaculaire est plus susceptible d'être utilisé lorsque l'on parle à ses pairs, ses intimes et ses proches, et généralement à un petit nombre d'entre eux, plutôt qu'à un groupe de personnes. Parler à un plus grand groupe d'allocutaires, même quand il s'agit de ses pairs, ses intimes, ou ses proches, déclenche généralement un style de discours plus "protocolaire»- sans doute puisque ce genre de situation tend à être 
associé à des événements de parole plus structurés ayant lieu dans des contextes institutionnels formels, tels que l'école ou le lieu de travail. Cependant je reconnais naturellement que le style vernaculaire peut parfois émerger dans des situations plus formelles, par exemple si le locuteur veut créer un effet spécial ou dans le cadre d'une performance artistique (cf. Coupland, 2007).

\section{La quête du vernaculaire}

Dans la sociolinguistique variationniste, le vernaculaire est généralement considéré comme « l'objet d'étude le plus intéressant » (Trudgill, $2003: 141$ ), et certains ont même comparé la quête de ce style à la recherche légendaire du Saint Graal, représentée dans la littérature arthurienne du Moyen Âge (Bergs, 2005 : 17). Mais l'intérêt du vernaculaire comme type de discours dépend naturellement des objectifs du projet de recherche en question. ${ }^{4}$

Une des raisons pour lesquelles le vernaculaire a souvent été considéré comme l'idéal est peut-être simplement que, dès les débuts de la sociolinguistique variationniste, l'on a reconnu qu'il est rare et difficile à obtenir dans le cadre d'un entretien entre inconnus. Mais la raison principale est sans doute qu'il est considéré comme la variété « la plus régulière et systématique » de la langue (Trudgill, $2003: 141$ ).

Cette attitude rappelle la préférence en dialectologie traditionnelle pour la forme la plus « pure » d'un dialecte rural, tel qu'il est parlé par les informateurs âgés qui ont rarement voyagé au-delà de leur pays local. ${ }^{5}$ Mais que veut dire précisément l'assertion que le vernaculaire est la forme « la plus régulière et systématique » de la langue ? Pour Trudgill, c'est que ce style est « moins influencée par d'autres variétés et par des notions prescriptives de correction linguistique »: en d'autres termes, une fois qu'un locuteur se trouve dans une situation informelle, et qu'il commence à s'exprimer dans son vernaculaire, il est peu susceptible de passer à une utilisation plus fréquente de variantes standard (ou formelles), tant que restent inchangés les éléments clé de la situation, tels que l'allocutaire, d'autres participants (s'il y en a), et le sujet de conversation. En revanche, dans des situations moins détendues, l'emploi de variables sociolinguistiques par les locuteurs est plus imprévisible, en ce sens que l'on ne peut pas anticiper comment va réagir tel ou tel locuteur, et combien il va s'éloigner de son style vernaculaire, en termes quantitatifs. ${ }^{6}$

Un autre facteur qui fait que le vernaculaire est considéré comme un objet d'étude particulièrement intéressant est la conviction chez les sociolinguistes que c'est dans ce style qu'apparaissent pour la première fois de nombreuses innovations linguistiques. Cette croyance est une extension de l'hypothèse largement répandue (mais qui n'est pas incontestée) selon laquelle la langue parlée serait à l'avant-garde de la plupart des changements. Or, il existe certainement des exceptions à cette règle hypothétique, notamment en ce qui concerne les changements dont les locuteurs ont un niveau de conscience élevé. Mais si l'on accepte globalement cette hypothèse, on peut conclure que le style vernaculaire représente l'indication la plus fiable de l'état actuel non seulement de changements particuliers mais également du système linguistique dans son ensemble, notamment dans une perspective typologique (Coveney, 2002 : 278). Comme l'a souligné Deulofeu (1983), dans les études inter-linguistiques il faut comparer des choses comparables, et non pas, par exemple, la variété hautement codifiée d'une langue avec une variété vernaculaire non standardisée d'une autre. Étant donné que les locuteurs de toutes les langues possèdent un vernaculaire, mais pas forcément une variété standardisée, il est plus logique de faire des comparaisons entre les langues sur la base des styles vernaculaires de chacune.

Une troisième raison pour le statut spécial du style vernaculaire en sociolinguistique est la préférence parallèle pour la langue des groupes défavorisés au sein de la communauté, tels que les locuteurs d'origine populaire ou des minorités ethniques : à titre d'exemples, on peut citer les recherches menées par Laks sur le langage des adolescents d'origine populaire à Villejuif (1980, 1983), ou le projet récent sur le français parisien multiculturel (Gadet, 2013a, 2013b). La grande enquête sur le français de Montréal en 1971 constitue un autre exemple, car la communauté francophone de Montréal était encore à cette époque sous la domination anglophone, en termes économiques et politiques. Le français de groupes défavorisés est souvent riche en variantes non standard qui caractérisent également le style vernaculaire dans la communauté dans son ensemble - d'où la confusion fréquente chez les profanes entre " français familier » (un style de discours informel ou familier) et «français populaire» (le sociolecte du peuple). Mener des recherches sur le langage des groupes défavorisés est considéré par beaucoup comme un moyen de contrer les attitudes négatives envers leurs variétés et donc comme une contribution à la lutte contre l'insécurité et les préjugés linguistiques dont ces groupes sont victimes. En termes bourdieusiens, on parle de domination linguistique (Encrevé, 2004). Les recherches sur le langage des groupes défavorisés sont donc motivées, en partie au moins, par une volonté de réduire les inégalités. À cet égard, Poplack \& St-Amand (2007) ont parlé de la " primauté du vernaculaire » en sociolinguistique.

Malgré l'accent mis sur le vernaculaire en sociolinguistique variationniste, il ne faut pas oublier que certaines variantes, en phonologie et en morphosyntaxe, ont été étudiées surtout dans des styles de parole plus formels, et cela pour la bonne raison qu'elles ne s'emploient que dans un discours plus ou moins 
« protocolaire » : c'est le cas, par exemple, de l'utilisation de l'on comme alternative à on, étudiée par Coveney (2004) dans les journaux télévisés, et de la liaison facultative sans enchainement, analysée par Encrevé (1983, $1988)$ et Laks $(2009,2014)$ dans le discours politique diffusé à la radio ou à la télévision.

\title{
3. La comparabilité des corpus
}

L'opportunité d'utiliser des données vernaculaires pour assurer la validité des comparaisons entre les langues, qui a été évoqué plus haut, a un parallèle dans l'étude du changement linguistique, que ce soit historique ou contemporain. Écrivant sur l'augmentation dans l'emploi de going to comme marqueur du futur dans les corpus de l'anglais écrit, Mair (1997: 1541) a affirmé :

\begin{abstract}
"Afin de comprendre la nature de cette augmentation à court terme, nous devons nous rappeler que la fréquence de "going to " dépend du type de texte [...] et que nous n'avons pas affaire à un corpus représentatif de la langue prise dans son ensemble, mais plutôt à un type spécifique d'échantillon, la langue de la presse, qui est une variété écrite publiée d'un niveau de formalité assez élevé. La propagation rapide de "going to » dans cette variété n'est donc pas un signe de changement grammatical, mais d'une évolution stylistique qui peut être documentée également de nombreuses autres façons : le rétrécissement de l'écart entre les normes de l'anglais parlé et de l'anglais écrit, la "familiarisation » [A.C : en anglais, "colloquialization"] de l'écriture anglaise, qui a eu lieu au cours des trente dernières années ».
\end{abstract}

En linguistique de corpus on appelle ce problème « l'évolution des genres » (Leech \& Smith, 2005). Lorsque l'on compare des textes de différentes périodes du XXe siècle, dans le but d'identifier des changements grammaticaux (ou autres), il faut garder à l'esprit qu'avec le passage du temps les documents publiés - en français et en anglais tout au moins - sont devenus plus ouverts à des formes linguistiques familières. ${ }^{7}$ Or, lorsqu'on constate une différence quantitative entre des échantillons de textes de deux périodes (ou plus), cette différence représente-t-elle un véritable changement linguistique en cours, ou plutôt un changement dans les attitudes concernant le niveau de langue qu'il convient d'employer dans tel ou tel genre, autrement dit un changement de «préférence stylistique »? Le même problème se pose en sociolinguistique variationniste : lorsque l'on compare deux échantillons de discours non vernaculaire, que ce soit d'époques différentes ou de groupes sociaux différents, il est primordial d'envisager la possibilité que les deux groupes de locuteurs aient pu parler dans des styles différents - l'un dans un style très informel, l'autre dans un style plutôt formel.

\section{La barrière du vouvoiement}

Un facteur déterminant en ce qui concerne la variation stylistique - et donc pour l'émergence du vernaculaire est le choix entre le vouvoiement et le tutoiement. C'est vrai dans toute situation de parole, y compris dans les entretiens sociolinguistiques, où ce choix reflète fidèlement le rapport entre enquêteur et informateur. Dans les entretiens menés en anglais, il est facile pour les interlocuteurs d'éviter complètement le choix potentiellement délicat d'un terme d'adresse approprié, puisque l'utilisation de ces formes est facultative, mais en français le choix entre $t u$ et vous est quasi-obligatoire, à moins de diminuer sérieusement la communication. La «barrière $\mathrm{du}$ vouvoiement » (pour reprendre une expression utilisée par André Martinet ${ }^{8}$ est sans doute un problème plus important pour le travail de terrain sociolinguistique en France qu'au Québec, ou dans plusieurs autres pays où l'on connait une distinction du type tu-vous, puisque le vouvoiement réciproque reste en France le modèle d'adresse normal entre adultes inconnus. Or, chose étonnante, la question de l'emploi de tu et vous dans les entretiens sociolinguistiques a été discutée très peu, à l'exception de quelques chercheurs québécois et anglophones tels que Thibault \& Daveluy (1989), Thibault (1991), Coveney (2003, 2010) et Beeching (2012).

Tableau 1. Le nombre d'interviewés utilisant tu ou vous, pour s'adresser à leur interlocuteur dans trois enquêtes sociolinguistiques.

\begin{tabular}{|l|l|l|l|}
\hline Pronom d'adresse utilisé & Montréal 1971 & Tours 1976 & Picardie (Somme) 1982 \\
\hline Vous & $* 79$ & 103 & $* * 6$ \\
\hline vous et $t u$ & 12 & 0 & 0 \\
\hline
\end{tabular}




\begin{tabular}{|l|l|l|l|}
\hline$T u$ & 28 & 0 & 22 \\
\hline Aucun & 1 & 0 & 2 \\
\hline Total & 120 & 103 & 30 \\
\hline
\end{tabular}

Notes : * Dont 54 femmes et 25 hommes. ** Dont 5 femmes. (Sources : Sankoff et alii, 1976 ; Ashby, 1981,

1992 ; Coveney, 2003. (Tableau adapté de Coveney, 2003.)

Jusqu'au milieu des années 1960, le tutoiement était moins répandu au Québec qu'en France : auparavant de nombreux enfants québécois vouvoyaient leurs grands-parents, et parfois même leurs parents, comme l'a démontré l'enquête de Lambert (1967). Dès 1970, cependant, un changement significatif s'est produit au Québec, et cela s'est reflété dans l'utilisation des pronoms d'adresse dans le corpus CedergrenSankoff (Montréal) de 1971. Le tableau 1 montre le nombre d'informateurs utilisant vous, tu, les deux ou aucun, pour s'adresser à l'intervieweur dans trois enquêtes sociolinguistiques : Montréal 1971, Tours 1976 (Ashby) et Picardie 1982 (Coveney). Cela nous montre que 28 des 120 personnes interrogées à Montréal ont utilisé le tutoiement, et douze autres ont employé à la fois tu et vous. Dans une des premières analyses de ses entretiens à Montréal, G. Sankoff (1973 : 57) a commenté quelques différences interpersonnelles dans deux souséchantillons du corpus : sur quatorze locuteurs âgés de plus de 50 ans, treize ont choisi vous comme pronom d'adresse, et l'autre a employé à la fois vous et $t u$; en revanche, parmi quatorze informateurs plus jeunes (15 à 25 ans), seulement quatre ont utilisé vous exclusivement, un a employé les deux pronoms et les neuf autres ont opté pour le tutoiement. Dans la mesure où le tutoiement implique souvent un discours généralement informel, ces résultats indiqueraient que les enquêteurs de Montréal ont probablement réussi à susciter chez les informateurs plus jeunes un style de parole assez détendu. Il est bien entendu significatif que les cinq enquêteurs avaient environ 24 ans, et que les interviewées âgés de 15 à 25 ans s'adressaient donc à quelqu'un qui était plus ou moins un pair.

Le tableau 1 révèle un contraste frappant entre les enquêtes à Tours et en Picardie à l'égard de l'utilisation du pronom d'adresse : alors que, dans la première, le vouvoiement a été systématique, il a été préféré par seulement six des enquêtés picards. Même si Ashby et moi étions tous les deux confrontés à un même problème en tant que chercheurs anglophones souhaitant réaliser des entretiens en France, nous avons choisi des démarches méthodologiques très différentes. Pour sa part, Ashby a privilégié la représentativité sociale en interviewant un échantillon stratifié selon l'âge, le sexe et l'origine sociale. En revanche, j'ai préféré favoriser l'obtention de données relativement proches du vernaculaire, en réalisant les entretiens auprès d'une trentaine d'adultes travaillant dans des colonies de vacances, un milieu que je connaissais déjà assez bien, à force d'avoir travaillé plusieurs fois dans des colonies de vacances et des stages de formation d'animateurs. Pendant le travail sur le terrain, j'ai visité chaque colonie pendant plusieurs jours, ce qui faisait que je n'étais pas totalement inconnu de mes informateurs au moment de l'entretien. Étant donné également que le tutoiement réciproque était en général la norme dans les colonies de vacances, il n'est pas surprenant que seulement six des enquêtés picards ont choisi le vouvoiement pendant leur entretien : pour cinq d'entre eux, ce choix s'expliquait sans doute par une plus grande distance sociale entre enquêteur et enquêtée, puisqu'il s'agissait de femmes ayant un écart d'âge assez important par rapport à moi. Deux autres informateurs ont simplement évité d'employer un pronom d'adresse au cours de l'entretien. Ces exceptions à la norme communautaire de tutoiement réciproque peuvent être interprétées en termes de degrés variables de " solidarité ", notion invoquée par Brown et Gilman (1960), dans leur étude classique des pronoms d'adresse, mais aujourd'hui on préfère le terme de " distance sociale », qui parait plus transparente.

Tableau 2. La fréquence de tu et vous dans trois corpus français.

\begin{tabular}{|l|l|l|l|}
\hline Corpus & $t u$ & vous & Occurrences de $t u$ et vous \\
\hline ESLO-1 (1969) & $1.6 \%$ & $98.4 \%$ & 6,319 \\
\hline Corpus de Bristol (c.1988) & $14.2 \%$ & $85.8 \%$ & 2,245 \\
\hline CRFP (2002) & $38 \%$ & $62 \%$ & 2,582 \\
\hline
\end{tabular}

(Adapté de Beeching, 2012.)

L'utilisation de tu et vous a également été analysée par Beeching (2012), dans trois autres corpus de français parlé : le Corpus de Référence du Français Parlé (un corpus de près de 290,000 mots, créé en 2002) son propre corpus de Bristol, composé d'interactions anonymes recueillies au cours des années 1980, et totalisant environ 155,000 mots, et enfin un sous-échantillon d'environ 300,000 mots du premier corpus 
d'Orléans (ESLO-1). ${ }^{10}$ À la différence des corpus variationnistes, le CRFP et le Corpus de Bristol comprennent des enregistrements de locuteurs venant de plusieurs régions différentes. Le tableau 2 ne montre pas le nombre de locuteurs employant $t u$ ou vous, mais plutôt la fréquence globale de ces deux pronoms dans chacun des trois corpus, y compris apparemment les occurrences produites par les enquêteurs. Dans le tableau 2, j'ai converti les chiffres bruts en fréquences relatives, pour faciliter la comparaison, et ces fréquences montrent que, dans les entretiens d'ESLO-1, le vouvoiement a été massivement préféré au tutoiement. (Il est probable que les quelques occurrences de $t u$ ont été produites, soit dans le discours direct, soit dans des paroles adressées à une tierce personne.) Selon Beeching, les différences entre les trois corpus reflètent peut-être une augmentation générale du tutoiement en France entre 1969 et 2002, et elle constate que cette interprétation est compatible avec les différences d'âge dans le CRFP pour l'utilisation de certains lexèmes désignés dans les dictionnaires comme « familiers », tels que bagnole, balade et bosser. Cependant, il est important également de noter que les trois corpus ont été enregistrés dans des contextes différents, et ne sont pas strictement comparables pour les fins d'une étude de changement en temps réel : par exemple, le corpus de Bristol (nommé ainsi par Beeching d'après son lieu de travail) comprend des «entretiens conversationnels », enregistrés principalement dans différents campings à travers la France, et qui, par conséquent, étaient moins structurés que les entretiens de ESLO-1.

J'ai insisté sur le lien entre le choix de pronom d'adresse ${ }^{11}$ et la formalité relative du discours produit par les locuteurs dans tel ou tel corpus, mais il reste à voir quelle est la force de cette corrélation. Peut-on caractériser le style vernaculaire en français parlé comme le «code $t u$ », en contraste avec le «code vous »? À mes yeux, une telle dichotomie ne représenterait rien de plus qu'une variante terminologique du « modèle diglossique du français ", qui, à mon avis, ne reflète pas la réalité du continuum de variation que l'on trouve en français parlé (Coveney, 2011). Mais existe-t-il une corrélation positive moins forte entre le vernaculaire et le tutoiement ? Certes le tutoiement n'entraine pas nécessairement l'utilisation du style vernaculaire (c'est-à-dire, l'emploi par un individu de son niveau maximal des variantes informelles ou non standard qui sont à sa disposition), mais il est probablement vrai que, pour l'immense majorité des locuteurs, le tutoiement est une condition nécessaire, mais non suffisante, pour l'émergence du style vernaculaire - ce qui impliquerait que le style vernaculaire est pragmatiquement incongru (et inexistant) aux côtés du vouvoiement dans le même discours.

Cette contrainte hypothétique peut être formulée comme suit :

(1) La contrainte des deux $\mathrm{V}$ : \# style vernaculaire + vouvoiement.

Il faut préciser que cette contrainte empêcherait, non pas la cooccurrence de quelques formes vernaculaires avec le vouvoiement, mais plutôt le vouvoiement avec le maximum statistique de ces formes, pour l'individu en question. Il est clair également que la contrainte que je propose ne peut pas tenir pour des locuteurs qui ne tutoieraient jamais personne, mais il est probable que de telles personnes sont extrêmement rares aujourd'hui. ${ }^{12}$ Compte tenu de la définition quantitative du style vernaculaire que j'ai proposée, l'on pourrait tester cette hypothèse sur des corpus de français parlé : existe-t-il des locuteurs qui combinent le vouvoiement avec l'emploi catégorique (ou même quasi-catégorique) de plusieurs variantes vernaculaires ?

\section{L'impact de la situation d'entretien sur la fréquence de certaines variables grammaticales}

En parallèle avec le besoin sociolinguistique d'obtenir ce que Labov a appelé " good data » - et surtout des données proches du style vernaculaire - il existe également la nécessité d'enregistrer un échantillon de locuteurs à peu près représentatif de la communauté linguistique en question. Et pour ce faire, la plupart des chercheurs variationnistes ont eu recours à l'entretien sociolinguistique, étant donné qu'il offre la possibilité d'obtenir environ une heure de discours de chaque informateur, en un temps relativement limité. Mais dans l'étude quantitative de la variation grammaticale, il est bien connu que l'on doit aussi souvent faire face au problème de la rareté de certaines variables grammaticales, notamment - mais pas exclusivement - dans les entretiens sociolinguistiques. Considérons maintenant l'impact de la dynamique interpersonnelle de l'entretien sur deux domaines de la grammaire : les pronoms sujets indéfinis et les structures syntaxiques associées aux actes de parole directifs et expressifs, notamment les interrogatives directes et les phrases négatives emphatiques.

Graphique 1. L'emploi par trente locuteurs de deux variantes grammaticales : on indéfini (en variation avectu/vous) et le maintien de ne (Corpus de Picardie (Source : Coveney, 2009)) 


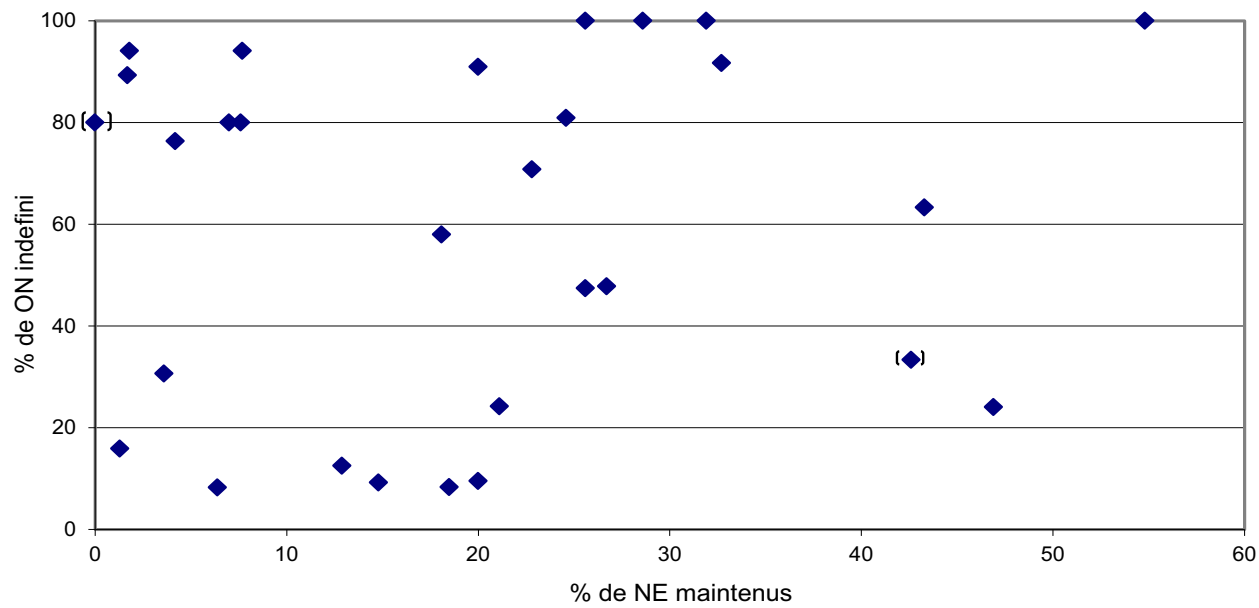

La variation entre on et $t u$ (ou vous) en tant que pronoms sujets indéfinis est illustrée dans les exemples suivants, tirés du corpus de Picardie :

(2) tu sais de euh - le plâtre des chirurgiens quoi quand on a un bras cassé. / [Locuteur 7] (3) la première fois que tu : $t u$ vois quelqu'un $t u$ lui dis « $\mathrm{s}-$ salut $» /$ [Locuteur 25] (4) j'estime qu'à l'heure actuelle vous avez des animateurs / qui vont subir des stages de quinze jours de formation / et qui vont se dire « stagiaires ». / [Locuteur 11]

Dans mes recherches sur cette variable dans le corpus de Picardie, j'ai trouvé qu'un groupe de locuteurs jeunes (âgés de 17 à 28 ans) ont combiné un très faible taux de maintien de la particule négative ne (moins de 10\%) avec un taux d'emploi très élevé du pronom indéfini on, en préférence à la variante informelle tu/vous (Coveney, 2003, 2009). Alors que le faible taux de ne implique un style de parole plutôt détendu, celui du tu/vous indéfini indiquerait au contraire un style plus formel. Sur le Graphique 1, ce groupe de locuteurs est représenté à l'extrême gauche de la partie supérieure. Cette distribution de données quantitatives est surprenante puisque, toutes choses étant égales par ailleurs, l'on s'attendrait à voir les locuteurs se comporter de manière similaire pour différentes variables - ce qui est effectivement le cas pour la majorité des informateurs sur le Graphique 1.

Tableau 3. Fréquence d'emploi de on comme pronom indéfini (en variation avec $t u$ ou vous) par quatre groupes de locuteurs ayant des fréquences d'emploi différentes d'un pronom d'adresse (tu ou vous) pendant l'entretien (Corpus de Picardie).

\begin{tabular}{|l|l|l|}
\hline $\begin{array}{l}\text { Groupes de locuteurs } \\
\text { (N de } t u / \text { vous } \\
\text { pour } 5,000 \text { mots })\end{array}$ & $\begin{array}{l}\text { Nombre de locuteurs } \\
\text { dans le groupe }\end{array}$ & $\begin{array}{l}\text { Fréquence moyenne de } \\
\text { on indéfini }\end{array}$ \\
\hline A $(0-1.2)$ & 6 & $95.7 \%$ \\
\hline B $(3.2-5.6)$ & 6 & $80.8 \%$ \\
\hline C $(8.6-13.5)$ & 7 & $53.7 \%$ \\
\hline D $(19.9$ ou plus $)$ & 8 & $21.5 \%$ \\
\hline
\end{tabular}

Note : Trois locuteurs sont exclus ici, car ils ont produit seulement 5, 3 et 0 occurrences de la variable $(o n \sim$ tu/vous), respectivement.

Afin d'explorer plus en détail le comportement exceptionnel de ce groupe de locuteurs, j'ai compté combien de fois chacun de mes informateurs a utilisé un pronom d'adresse (c'est-à-dire tu ou vous avec référence définie). Ensuite, pour faire des comparaisons interpersonnelles, j'ai calculé pour chaque individu le nombre d'occurrences du tu/vous défini pour 5,000 mots de leur discours. Étant donnée la corrélation entre ce 
chiffre et la fréquence d'emploi du pronom on comme indéfini, il s'est avéré que les locuteurs qui utilisaient moins souvent tu/vous comme pronom d'adresse avaient également une forte tendance à éviter tu/vous comme pronom indéfini, et montraient ainsi une préférence massive pour on comme pronom indéfini. D'autre part, ceux qui ont employé assez souvent un pronom d'adresse (que ce soit $t u$ ou vous), utilisaient souvent le même pronom pour la référence indéfinie. Cette distribution des faits peut être représentée clairement si l'on compare les scores moyens de quatre groupes d'informateurs, présentés dans le tableau 3. Les locuteurs ont été placés dans l'un des quatre groupes (A, B, C, D), selon le nombre d'occurrences de tu/vous comme pronom d'adresse qu'ils ont produites tous les 5,000 mots. ${ }^{13}$ Le groupe A, qui a produit moins de 1,3 occurrences du $t u$ défini tous les 5,000 mots, a montré une très forte tendance à éviter le $t u$ indéfini, car sa fréquence moyenne pour on est de $95,7 \%$. En revanche, le groupe D a produit assez souvent des occurrences du tu/vous défini, avec un taux de 19,9 ou plus pour 5,000 mots. ${ }^{14}$ Et lorsqu'ils ont dû choisir un pronom sujet indéfini, ils ont opté principalement pour $t u$ ou vous, plutôt que on. Mon interprétation de ces distributions est que les informateurs du groupe A, qui me tutoyaient tous, se sentaient néanmoins quelque peu réticents quand il s'agissait de produire des occurrences de $t u$ au cours de l'entretien, et ont donc préféré éviter cette forme chaque fois que cela était possible, en raison peut-être d'un sentiment un peu incertain de la distance sociale entre eux et moi, malgré le fait que le tutoiement réciproque entre nous était apparemment bien établi.

Étant donnée la signification sociale du choix entre vouvoiement et tutoiement, on peut dire que chaque occurrence de $t u$ ou vous a une fonction phatique importante, et sert en partie à exprimer la perception qu'a le locuteur de la distance sociale entre lui-même et son allocutaire. Le groupe A du tableau 3 comprend quatre des plus jeunes informateurs âgés de 17 à 20 ans (deux femmes, deux hommes), ainsi que deux femmes d'environ le même âge que l'enquêteur (27 ans). À la différence de ce groupe, les huit informateurs du groupe D semblaient plus à l'aise avec leur choix de pronom d'adresse : ce groupe comptait trois femmes âgées de 50 à 60 ans (qui ont utilisé vous, comme on pouvait s'y attendre), mais également les deux locuteurs les plus loquaces (deux hommes âgés d'environ trente-cinq ans, qui étaient tous les deux directeurs de colonie), plus trois autres informateurs âgés de 17 à 20 ans. Puisque le tu/vous indéfini était déjà, au moment de l'enquête, bien établi dans les styles informels du français parlé, ma conclusion est que l'évitement de ces pronoms par certains informateurs s'explique par leur réaction à la situation de l'entretien, et non pas à une différenciation sociolinguistique proprement dite. En ce qui concerne la variation entre on et tu/vous comme pronoms indéfinis, il est clair que les entretiens n'ont pas réussi à faire apparaitre le style vernaculaire - ou même quasivernaculaire - de ce groupe de locuteurs, et qu'il faudrait obtenir plutôt un type de discours plus spontané, dans lequel tous les locuteurs sont plus à l'aise avec leur choix de pronom d'adresse. ${ }^{15}$

Il est important de noter qu'aucun informateur du Corpus de Picardie n'a employé au cours de son entretien le vouvoiement pour l'adresse en même temps que le $t u$ comme pronom indéfini (ou dans les marqueurs de discours tels que $t u$ vois et $t u$ sais). Cela contraste nettement avec ce qui a été trouvé dans les entretiens du corpus Cedergren-Sankoff, enregistré à Montréal en 1971. À cet égard, G. Sankoff (1973 : 57) a comparé un sous-échantillon de quatorze informateurs âgés de 50 ans ou plus avec un groupe de quatorze locuteurs plus jeunes (de 15 à 25 ans). ${ }^{16}$ Quatre membres du groupe plus âgé ont utilisé le tu indéfini (en variation avec on), même s'ils ont également vouvoyé l'enquêteur, soit systématiquement, soit de manière variable. Parmi les plus jeunes, l'utilisation du $t u$ indéfini était beaucoup plus fréquente : douze des quatorze locuteurs ont eu un taux de $70 \%$ pour le $t u$ indéfini, et trois d'entre eux ont systématiquement vouvoyé l'enquêteur. Il semble probable que ce type d'alternance entre $t u$ et vous - vous pour l'adresse, $t u$ pour la référence indéfinie - se produise également parfois dans les variétés européennes du français, bien qu'il soit absent du Corpus de Picardie.

Un autre domaine grammatical où la fréquence est particulièrement affectée par l'entretien concerne les structures syntaxiques associées aux fonctions directives et expressives. Selon Cheshire (1996), la négation et l'interrogation sont deux contextes « proéminents » du point de vue socio-pragmatique, dans le sens où elles sont souvent liées à l'interaction face-à-face : dans la langue parlée, les interrogatives demandent souvent une réponse immédiate de la part de l'allocutaire, tandis que la négation sert fréquemment à contredire une proposition qui vient d'être exprimée, soit explicitement, soit implicitement. Or, l'omission facultative de la particule négative ne est une des variables grammaticales les plus fréquentes dans le français parlé, et cette fréquence n'est pas spécialement influencée par la situation d'entretien. En revanche, dans les corpus d'entretiens enregistrés en France, il y a une pénurie frappante de deux formes de ce que l'on pourrait appeler la « négation expressive ou emphatique ». La première consiste en l'emploi du mot pas pour renforcer un autre complément négatif, une structure traditionnellement associée au français "populaire » et à certains dialectes d'Ö̈l. ${ }^{17}$ L'exemple suivant est l'unique occurrence de cette structure dans le Corpus de Picardie :

(5) dans le bilan général j'ai pas eu aucune difficulté. / [Locuteur 23] La deuxième forme de négation expressive comporte l'antéposition d'un complément négatif (le plus souvent pas, mais parfois plus), renforcé par l'adverbe même - une structure qui est attestée dans le français parisien multiculturel: 
(6) elle, elle arrive en retard / nous, on est là à poireauter / même pas elle s'excuse ! (Dekhissi, $2013: 58$ ). ${ }^{18}$ Il est possible que cette forme de négation emphatique soit une innovation. ${ }^{19}$ En tout cas, il est quasiment certain que les deux structures négatives sont socialement différenciées, mais leur rareté dans les entretiens fait que, jusqu'à présent, ni l'une ni l'autre n'a fait l'objet d'une étudie quantitative pour le français de France (sauf erreur de ma part). ${ }^{20}$ La rareté (ou l'inexistence) de l'antéposition de même pas dans les entretiens pourrait s'expliquer en partie par le fait que cette structure soit une innovation, et qu'elle ne soit pas encore très répandue en France. Mais la faible fréquence, dans les corpus français, de l'autre type de négation expressive («pas aucun ») semble attribuable plutôt à la nature socio-pragmatique de l'entretien, et au manque relatif d'expressivité dans ce type de discours.

Le type de discours affecte la fréquence des interrogatives encore plus que celle des phrases négatives, et, toutes choses égales par ailleurs, l'on ne s'attend pas à rencontrer beaucoup de questions dans le discours de personnes interviewées. Dans le Corpus de Picardie, j'ai néanmoins trouvé 180 occurrences de l'interrogation totale (mis à part les marqueurs discursifs tels que voyez-vous ? et tu vois ?), et 122 cas de l'interrogation partielle. Certaines de ces interrogatives ont été adressées à une tierce personne lors d'une interruption spontanée dans l'entretien proprement dit, mais la majorité en étaient des questions posées à l'enquêteur (surtout des interrogatives totales) ou bien des interrogatives utilisées pour d'autres fonctions communicatives, telles que des citations et des questions auto-adressées. Ces quantités d'occurrences ont permis une analyse qualitative des interrogatives, notamment concernant la dimension pragmatique et les contraintes linguistiques catégoriques, mais elles étaient trop faibles pour une étude quantitative approfondie des différences interpersonnelles ou des contraintes variables.

Comme l'ont constaté Thibault et Vincent (1990:46-7), les demandes, les ordres et les signes d'agressivité sont extrêmement rares dans les entretiens sociolinguistiques. D'une part, cela a l'avantage de favoriser une interaction harmonieuse et agréable, mais de l'autre il rend moins probable que l'informateur produise un langage expressif, avec des structures potentiellement variables telles que les questions rhétoriques et les phrases exclamatives. Dans le Corpus de Picardie, j'ai identifié 55 sous-catégories de fonction communicative exprimées par une interrogative directe. Mais seulement neuf des 302 propositions interrogatives directes étaient des questions rhétoriques, une proportion beaucoup plus faible que ce que l'on trouve dans certains autres types de discours. Qui plus est, aucune n'était ce qu'on peut appeler une Question Rhétorique Conflictuelle, servant à critiquer l'allocutaire ou une tierce personne, comme dans les exemples suivants, tirés du film Raï (1995) :

(7) Papa, pourquoi tu la laisses sortir comme ça ?! / Pour qu'elle aille trainer / c'est ça ? (Dekhissi, 2015 : 10) De même, les phrases exclamatives sont pratiquement inexistantes dans le Corpus de Picardie, alors qu'elles sont assez fréquentes dans le corpus filmique étudié par Dekhissi (à paraitre).

\section{Le paradoxe variationniste}

De toute évidence, il existe dans les recherches variationnistes un conflit quadridimensionnel impliquant la nécessité d'avoir un échantillon démographique représentatif, un discours spontané proche du vernaculaire, et un nombre suffisant d'occurrences de la variable en question pour une étude quantitative - tout cela en un temps limité pour réaliser le travail sur le terrain. Ce conflit peut être formulé comme un " paradoxe variationniste » (en parallèle avec le célèbre paradoxe de l'observateur, énoncé par Labov, et qui découle de l'effet de l'observateur dans différentes disciplines scientifiques) :

(8) Le paradoxe variationniste : Dans la plupart des recherches variationnistes, l'objectif idéal est d'enregistrer, en un temps assez limité, une quantité importante de discours en style vernaculaire chez un échantillon représentatif de la communauté linguistique. Le contexte le plus pratique pour obtenir une telle quantité de données auprès d'un tel échantillon est l'entretien sociolinguistique, qui, par définition, est une situation impliquant deux inconnus et qui, par conséquent, exclut le style vernaculaire.

Ce paradoxe peut-il être résolu? Pour un projet à très grande échelle, l'on pourrait envisager la réalisation de deux entretiens (ou plus) avec chaque informateur, étalés sur plusieurs semaines ou mois. À force de créer des liens d'amitié avec ses informateurs, une équipe d'enquêteurs exceptionnellement doués pourrait ainsi réussir à établir une relation d'amitié avec chacun des informateurs, ce qui rendrait plus probable l'émergence du style vernaculaire dans le contexte de l'entretien. Une alternative plus pratique - surtout pour les projets ayant des ressources plus modestes - serait qu'à la suite de l'entretien, l'informateur soit invité à enregistrer lui-même ses conversations sur une période de plusieurs jours, y compris des interactions en style vernaculaire avec des pairs et des intimes. C'est ce qu'ont fait précisément Diane Vincent et ses collègues, au cours de la troisième enquête sociolinguistique sur Montréal, mais pour seulement quatre informateurs (Vincent, 
Laforest \& Martel, 1995). Une autre possibilité est la conversation entre pairs, pour laquelle on demande à un informateur de choisir un ou deux amis proches comme interlocuteurs, et ensuite de se faire enregistrer en conversation. Cette méthode a été employée avec succès par Armstrong dans son enquête chez des lycéens en Lorraine $(1993,2002)$, et cela lui a permis de contraster ce style proche du vernaculaire avec un entretien sociolinguistique qu'il a réalisé avec les mêmes informateurs.

\section{Références bibliographiques}

Armstrong, Nigel. 1993. A Study of phonological variation in French secondary school pupils. University of Newcastle upon Tyne, PhD thesis.

Armstrong, Nigel. 2002. Variable deletion of French ne : a cross-stylistic perspective. Language Sciences, 24 : $153-173$

Armstrong, Nigel \& Tim Pooley. 2010. Social and linguistic change in European French. London : Palgrave Macmillan.

Ashby, William J. 1981. The loss of the negative particle ne in French : a syntactic change in progress. Language, $57:$ 674-687.

Ashby, William J. 1992. The variable use of on versus tu/vous for indefinite reference in spoken French. Journal of French Language Studies, 2 : 135-157.

Beeching, Kate. 2012. Sociolinguistic aspects of lexical variation in French. In: Tim Pooley \& Dominique Lagorgette (eds.) On linguistic change in French: socio-historical approaches (Le changement linguistique en français : aspects socio-historiques). Chambéry : Presses Universitaires de Savoie. pp. 37-54.

Bell, Allan 1984. Language Style as Audience Design. Language in Society, 13 : 145-204.

Bell, Allan 2001. Back in style : reworking audience design. In : Penelope Eckert \& John R. Rickford (eds.) Style and Sociolinguistic Variation. Cambridge University Press. pp.139-69.

Bergs, Alexander. 2005. Social networks and historical sociolinguistics : studies in morphosyntactic variation in the Paston letters (1421-1503). Berlin : De Gruyter.

Brown, Roger \& Albert Gilman. 1960. The pronouns of power and solidarity. In : Thomas Sebeok (ed.) Style in language. Cambridge, MA. : MIT Press. pp. 253-276.

Burnett, Heather, Mireille Tremblay \& Hélène Blondeau. 2015. The Variable Grammar of Negative Concord in Montréal French. University of Pennsylvania Working Papers in Linguistics, 21/2, Article 3. Disponible à : http ://repository.upenn.edu/pwpl/vol21/iss2/3.

Cheshire, Jenny. 1996. Syntactic variation and the concept of prominence. In : Juhani Klemola, Merja Kytö \& Matti Rissanen. (eds.) Speech past and present : studies in English dialectology in memory of Ossi Ihalainen. Frankfurt : Lang. pp. 1-17.

Klemola, Juhani, "Speech Past and Present. Studies in English Dialectology in Memory of Ossi Ihalainen." (1996).

Coupland, Nikolas. 1980. Style-shifting in a Cardiff work setting. Language in Society, 9 : 1-12.

Coupland, Nikolas. 2007. Style : Language variation and identity. Cambridge University Press.

Coveney, Aidan. 2002. Variability in Spoken French : interrogation and negation. ( ${ }^{\mathrm{e}}$ édition). Bristol : Intellect.

Coveney, Aidan 2003. "Anything you can do, $t u$ can do better »: $t u$ and vous as substitutes for indefinite on in French. Journal of Sociolinguistics, $7: 164-191$.

Coveney, Aidan. 2004. The alternation between l'on and on in spoken French. Journal of French Language Studies, 14 : 91-112.

Coveney, Aidan. 2009. On pour tous et $t u$ pour on : tu et vous comme pronoms indéfinis. In :

Bert Peeters \& Nathalie Ramière (dirs.) Tu ou Vous : l'embarras du choix. Limoges : Editions Lambert-Lucas. pp. 253-285.

Coveney, Aidan. 2010. Vouvoiement and tutoiement : sociolinguistic reflections. Journal of French Language Studies, 20 : 127-150.

Coveney, Aidan. 2011. A language divided against itself? Diglossia, code-switching and variation in French. In : France Martineau \& Terry Nadasdi (dirs.) Le français en contact : Hommages à Raymond Mougeon. Québec : Presses de l'Université Laval. pp. 51-85.

Dekhissi, Laurie. 2013. Variation syntaxique dans le français multiculturel du cinéma de banlieue. Université d'Exeter (G-B), thèse de doctorat.

Dekhissi, Laurie. 2015. "Qu'est-ce t'as été te mêler de ça?! » : Une « nouvelle » structure pour les questions rhétoriques conflictuelles. Journal of French Language Studies, FirstView: 1-20.

Dekhissi, Laurie. (à paraitre). De «Comme t'es jalouse ! » à «Comment t'es belle !» : Variation dans l'emploi des exclamatives en français parlé.

Deulofeu, José. 1983. L'étude des langues parlées et la typologie des langues. Recherches sur le français parlé, $5: 103-123$. 
Encrevé, Pierre. 1983. La liaison sans enchaînement. Actes de la recherche en sciences sociales, 46 : 39-66.

Encrevé, Pierre. 1988. La Liaison avec et sans enchaînement : Phonologie tridimensionnelle et usage du français. Paris : Seuil.

Encrevé, Pierre. 2004. Langue et domination. In : Louis Pinto, Gisèle Sapiro \& Patrick Champagne (dirs.) Pierre Bourdieu, sociologue. Paris : Fayard. pp. 289-305.

Encrevé, Pierre. 2009. Méthodes en linguistique synchronique. La linguistique, 45/1 : 37-60.

Fonseca-Greber, Bonnie \& Linda R. Waugh. 2003. On the radical difference between the subject personal pronouns in written and spoken European French. In : Pepi Leistyna \& Charles F. Meyer. (eds.) Corpus Analysis : Language Structure and Language Use. Amsterdam : Rodopi. pp. 225-240.

Gadet, Françoise. 2013a. Un nouveau corpus recueilli dans la région parisienne. Langage et société, 143 : 111 117.

Gadet, Françoise. 2013b. Collecting a new corpus in the Paris area : Intertwining methodological and sociolinguistic reflections. In : David Hornsby \& Mari C. Jones (eds.). Language and Social Structure in Urban France. Oxford : Legenda. pp. 162-171.

Gadet, Françoise \& Emmanuelle Guerin. 2012. Quelles données pour cerner quels objets ? Exemples de « parlers jeunes ». Séminaire doctoral du laboratoire MoDyCo, de l'Ecole doctorale 139 et de l'Ecole doctorale 180, UPOND, le 13 mars 2012. (Universié Paris-Ouest Nanterre la Défense). Disponible à :

http : :/www.modyco.fr/en/presentations-seminaire-modyco/605-2012-13mars-gadet-guerin-exemplierbiblio/file.html

Gadet, Françoise \& Philippe Hambye. 2014. Contact and ethnicity in « Youth Language » description : in search of specificity. In : Robert Nicolaï (ed.) Questioning Language Contact : Limits of Contact, Contact at its Limits. Leiden : Brill. pp. 183-216.

Labov, William. 1976. Sociolinguistique. Paris : Minuit. (Traduction par Alain Kihm de Sociolinguistic Patterns, 1972)

Laks, Bernard. 1980. Différenciation linguistique et différenciation sociale : quelques problèmes de sociolinguistiques française. Université de Paris VIII, thèse de 3ème Cycle.

Laks, Bernard. 1983. Langage et pratiques sociales : étude sociolinguistique d'un groupe d'adolescents. Actes de la recherche en sciences sociales, 46 : 73-97.

Laks, Bernard. 2009. Dynamiques de la liaison en français. In : Luc Baronian \& France Martineau (dirs.) Le français d'un continent à l'autre. Québec : Presses de l'Université Laval. pp. 237-267.

Laks, Bernard. 2014. Diachronie de la liaison en français contemporain : le cas de la parole publique (19992011). In : Jacques Durand, Gjert Kristoffersen, Bernard Laks \& Julie Peuvergne (dirs.) La phonologie du français: normes, périphéries, modélisation. Mélanges pour Chantal Lyche. Paris: Presses Universitaires de Paris Ouest. pp. 327-379.

Lambert, Wallace E. 1967. The use of $t u$ and vous as forms of address in French Canada : a pilot study. Journal of Verbal Learning and Verbal Behavior, 6/4 : 614-617.

Larrivée, Pierre. 2014. The continuity of the vernacular: The case of the evolution of negative doubling in French. In : Maj-Britt Mosegaard Hansen \& Jacqueline Visconti (eds.) The Diachrony of Negation. Amsterdam : Benjamins. pp. 235-256.

Leech, Geoffrey \& Nicholas Smith. 2005. Extending the possibilities of corpus-based research on English in the twentieth century : a prequel to LOB and FLOB. ICAME Journal, 29 : 83-98.

http ://www.lancaster.ac.uk/fass/doc_library/linguistics/leechg/leech_and_smith_2005.pdf

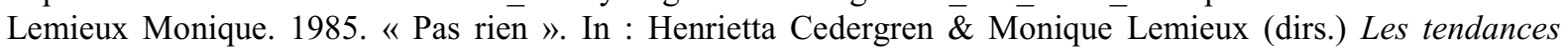 dynamiques du français parlé à Montréal, tome 2. Québec : Gouvernement du Québec. pp. 91-140.

Mair, Christian. 1997. The spread of the going-to-future in written English : A corpus-based investigation into language change in progress. In : Raymond Hickey \& Stanisław Puppel (eds.) Language History and Linguistic Modelling : A Festschrift for Jacek Fisiak on his 60th birthday. Berlin : Mouton de Gruyter. pp. 1537-1543.

Milroy, James. 1992. Linguistic variation and change. Oxford : Blackwell.

Poplack, Shana \& Anne St-Amand. 2007. A real-time window on 19th-century vernacular French : The Récits du français québécois d'autrefois. Language in Society, 36 : 707-734.

Robb, Graham. 2008. The Discovery of France. London : Pan Macmillan.

Sankoff, David, Gillian Sankoff, Suzanne Laberge \& Marjorie Topham. 1976. Méthodes d'échantillonnage et utilisation de l'ordinateur dans l'étude de la variation grammaticale. In : Henrietta Cedergren et alii (dirs.) La sociolinguistique au Québec. Montréal : Presses de l'Université du Québec.

Sankoff, Gillian. 1973. Above and beyond phonology in variable rules. In : Charles-James N. Bailey \& Roger Shuy (eds.) New ways of analyzing variation in English. Washington, D.C. : Georgetown University Press.

Sankoff, Gillian. 1980. The Social Life of Language. Philadelphia : University of Pennsylvania Press.

Thibault, Pierrette. 1991. La langue en mouvement : simplification, régularisation, restructuration. LINX, 25 : 79-92. 
Thibault, Pierrette \& Michelle Daveluy. 1989. Quelques traces du passage du temps dans le parler des Montréalais, 1971-1984. Language Variation and Change, 1/1 : 19-45.

Thibault, Pierrette \& Diane Vincent. 1990. Un corpus de français parlé. Québec : CIRAL, Université Laval.

Trudgill, Peter. 1986. Dialects in contact. Oxford : Blackwell.

Trudgill, Peter. 2003. A Glossary of Sociolinguistics. Edinburgh University Press.

Vincent, Diane, Marty Laforest et Guylaine Martel. 1995. Le corpus de Montréal 1995 : Adaptation de la méthodologie sociolinguistique pour l'analyse conversationnelle. Dialangue, 6 : 29-45.

\footnotetext{
${ }^{1}$ Traduction de A.C.

${ }^{2}$ Par « variables sociolinguistiques disponibles », j'entends celles qui sont régulièrement utilisées dans la communauté linguistique en question.
}

${ }^{3}$ En anglais, « Audience Design ».

${ }^{4}$ Si l'une des variantes à l'étude ne se produit que dans un style formel, le discours proche du vernaculaire montrera simplement l'utilisation catégorique de l'autre variante, sans différenciation sociale ni contraintes linguistiques.

${ }^{5}$ Selon Robb (2008 : 13), avant l'invention de bicyclettes bon marché, le monde connu, pour beaucoup d'habitants des régions rurales, avait un rayon de moins de vingt-quatre kilomètres.

${ }^{6}$ Les variables sociolinguistiques concernées ici sont, bien entendu, des « marqueurs » (celles qui sont sujettes à la variation stylistique, ainsi qu'à la stratification sociale), non pas des « indicateurs », dont l'emploi reste constant dans des styles différents.

${ }^{7}$ À cet égard, Armstrong \& Pooley (2010) emploient le terme "informalisation » plutôt que «familiarisation », car ils voient des parallèles entre le langage et d'autres aspects du comportement humain, tels l'habillement et la politesse.

${ }^{8}$ Selon Encrevé (2009), André Martinet avait employé l'expression « la barrière du vouvoiement » en 1964, en parlant de l'impossibilité d'un tutoiement réciproque entre lui-même, professeur à la Sorbonne, et son futur doctorant, Pierre Encrevé. Martinet avait toutefois proposé qu'ils s'adressent réciproquement avec leurs prénoms, plutôt qu'avec des termes plus formels.

${ }^{9}$ Le CRFP comprend le discours de 82 locuteurs venant d'une quarantaine de villes françaises. L'échantillon est à peu près équilibré pour le sexe, le niveau de scolarité et l'âge (de 18 ans jusqu'aux retraités). Voir:

http://sites.univ-provence.fr/ veronis/pdf/2004-presentation-crfp.pdf

${ }^{10}$ Voir http://bach.arts.kuleuven.be/elicop/ et http://eslo.huma-num.fr/

${ }^{11}$ Le choix entre tutoiement et vouvoiement implique bien entendu non seulement des pronoms sujets, mais également des verbes, des déterminants et des pronoms possessifs.

12 Selon Brown \& Gilman (1960), l'un de leurs informateurs français en 1957 était un jeune homme d'une famille aristocratique, qui a déclaré que la seule personne qu'il avait tutoyée au cours de sa vie était la vieille femme qui avait été sa gouvernante pendant son enfance. Brown et Gilman ont interprété ce témoignage en termes du modèle traditionnel de vouvoiement réciproque chez les aristocrates, qui avait donc exclu le tutoiement dans sa famille ou avec ses amis aristocratiques, alors que l'idéologie égalitaire de la société moderne l'avait bien entendu empêché d'employer le tutoiement non réciproque avec des interlocuteurs venant d'un milieu social « inférieur».

${ }^{13}$ Seules les occurrences de vous employé comme pronom singulier de politesse ont été incluses pour cette étude. Les cas de tu ou vous définis comprennent non seulement les occurrences des pronoms d'adresse, mais aussi l'utilisation de $t u$ ou vous dans certains marqueurs de discours, comme voyez-vous? et $t u$ vois ?

${ }^{14}$ Certains locuteurs du groupe D ont produit beaucoup plus d'occurrences de $t u$ ou vous défini que la moyenne de leur groupe. Les huit scores individuels de ce groupe étaient de 19.9, 24.9, 28.2, 28.6, 29.3, 37.0, 54.7 et 78.9 .

${ }^{15}$ Voir Fonseca-Greber \& Waugh (2003), pour une étude de ce type, à partir d'un corpus de conversations spontanées : vingt-sept locuteurs français ou suisses, originaires des classes intermédiaires, qui ont produit un taux global de $68 \%$ pour le $t u$ indéfini, à côté de $30.7 \%$ pour on et $1.3 \%$ pour vous.

${ }^{16}$ Ces détails ont été omis dans la version remaniée de l'article publiée dans G. Sankoff (1980). 
${ }^{17}$ Le statut stigmatisé de cette structure est comparable à celui de la « concorde négative » que l'on trouve dans beaucoup de variétés non standard de la langue anglaise.

${ }^{18}$ Cet exemple de l'antéposition de même pas s'est produit dans L'Esquive (2004), un film qui se déroule à Saint-Denis, une banlieue populaire de Paris.

${ }^{19}$ D'autres exemples de cette structure, dans le corpus du Français Parisien Multiculturel, sont cités par Gadet \& Guerin (2012), dont le suivant :

(9) même pas une meuf elle dit ça.

Un des exemples présentés par Gadet \& Guerin est produit en réponse à une question posée par l'enquêtrice, mais quant aux autres, il n'est pas clair s'ils ont été produits dans un entretien ou dans un contexte « écologique ». (Ailleurs, Gadet a mis en question la pertinence de termes tels que « multi-ethnolecte »: Gadet \& Hambye, 2014.)

${ }^{20}$ Par contre, pour le français québécois, il y a eu au moins deux études quantitatives de la double négation du type «pas aucun » (Lemieux, 1985 ; Burnett, Tremblay \& Blondeau, 2015), et, selon Larrivée (2014), cette structure - qu'il a nommée « redoublement négatif »- est bien plus fréquente au Québec qu’en France. 